\title{
Design and Implementation of an Interactive Service Robot
}

\author{
Belal Khawja Ashhad, Irin Sultana Bristy, Humayara Khatun, Kaphey Kalam, Ebad Zahir \\ Department of Electrical and Electronics Engineering, American International University-Bangladesh, Dhaka, \\ Bangladesh \\ Email: ashhadkhawja@gmail.com
}

Received October 2015

\begin{abstract}
A low cost service robot was designed and implemented specifically to be used in service industries e.g. hotels. This service robot can deliver a requested payload (towels, bed-sheets, drinks or toiletries) to a customer. As evident with modern technology, robots are assisting man in several avenues of the service industry. This designed service robot can propose an error free service, work around the clock and consume less time with requested tasks. The prototype can handle two commands received from two different rooms simultaneously. It has the ability to choose to always deliver to the closest room from its base first. Also, it has the function to carry two different types of payloads in two of its compartments and to lift up the desired payload ordered by the customer using a scissor lift system. The service robot also uses a touch screen to interact with the customer. The algorithm of the design ensures that the payload will always be delivered to the right customer in due time.
\end{abstract}

\section{Keywords}

Intelligent Automation, Interactive Robot, Consumer and Service Industry

\section{Introduction (History and Overview)}

The use of robots in Hotel service was first introduced in Aloft Hotel in Cupertino, California. The Robot named Botlr was created by Silicon Valley robotics company Savioke. It comes in a cylindrical shape with a basin and a lid on top. It can carry standard room service items like toiletries, water bottles, and newspapers, and can guide itself to a hotel room. It can also use the elevator. A staff dials in a room number and fills the top facing bin with the ordered service item. Botlr's intelligence is powered by software that its inventor says operates much like Microsoft Kinect's motion-sensing technology. Botlr can map its route by scanning as far as 40 feet ahead. Higher-resolution image sensors cue Botlr if unexpected obstacles, such as people or laundry carts on the move within a six-foot radius come on its way [1]. Although the cost of Botlr has never been revealed, looking at its features it is ought to be very expensive.

According to an online survey conducted to find interest of hotel customers in hotel service robots like SaviOne, it was found that [2]: 
1. Fifty-six percent of the people surveyed would like to have robotic room service.

2. $51 \%$ of the people surveyed would want a robot that can deliver room items.

3. Half of respondents age 25 - 34 would more willing to choose to stay in a hotel with robot than without one.

So, a low-cost and interactive service robot was designed which can deliver standard room service items required by the customer from room to room. The design for the prototype was initiated by first selecting the material. The choice of the material was influenced by the weight of the material and torque of the motor. Since the cost of the design needed to be minimized, a cheaper motor had to be selected. This consequently affected the size and speed of this humanoid robot. Future prototypes can be made faster/bigger if more money is invested on the motors.

A pair of Arduinos is used as the controlling unit. An Arduino UNO along with a GPRS module and a 2.8" TFT touch display are used for communication and interaction, and an Arduino MEGA with motors and sharp sensors are used for motion and sensing of the robot.

\section{Service Robot}

\subsection{System Layout}

Figure 1 shows the layout of the robot. The head of the Robot contains the Arduino UNO stacked with GPRS shield and 2.8” TFT Touch display, so that it is easier for the customer to interact with the system. The body has two compartments which can be filled with two different types of service items and the compartments can move vertically depending on the type of service item ordered. The compartment carrying that desired item will move up once that customer has verified the order. The base contains the other Arduino which controls the robot's motion as well as the motion of scissor lifts and sharp distance sensor. The distance sensor allows the robot to stop its motion if it detects any obstacle within a certain distance limit. The motor driver plays an important role in providing the required current to drive the motors as the Arduino doesn't have sufficient output current. The motors used are high torque $12 \mathrm{~V}$ DC-gear motors and smoothly supports the system. A 12-V LIPO (Lithium Polymer) battery is used for the design prototype.

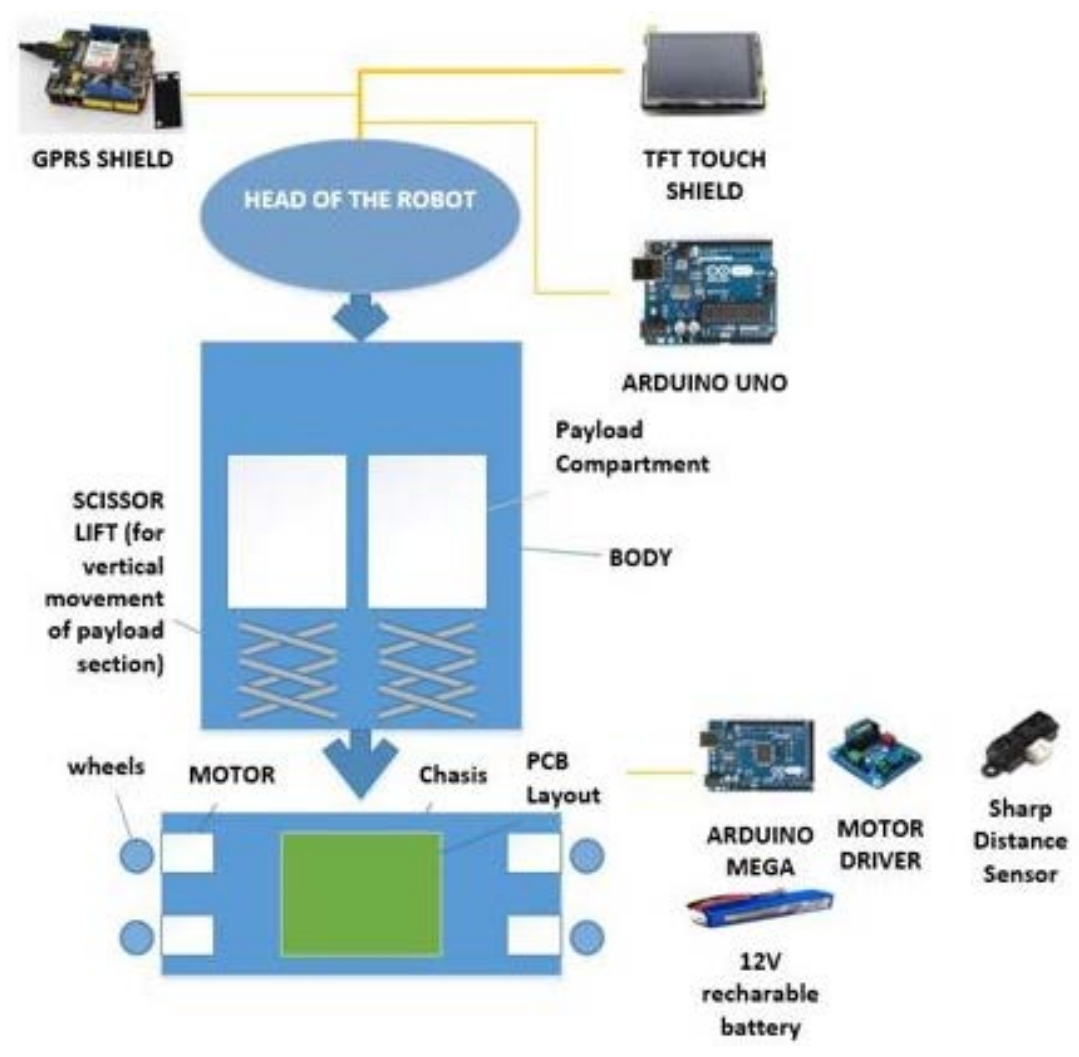

Figure 1. Layout of the robot. 


\subsection{Algorithm Flow Chart}

The Robot initially stays at its base position from where any customer walking in the lobby can walk up to it and interact with it. The operational process is shown in Figure 2. The touchscreen initializes with the standby menu, the customer can make calls to the RECEPTION, CAFE or the INFORMATION center of the hotel using the robot if needed. The robot will not receive any message unless it is on standby menu. When the first message/command will be received through the GPRS module and Arduino UNO using AT commands [3], the robot displays it on the screen in the format ROOM NO PAYLOAD TYPE; the robot once on that menu waits 15 seconds for another command and if there is no command Arduino Uno sends a signal to Arduino MEGA to initiate movement towards the designated room. Once the robot reaches the designated room Arduino MEGA sends a signal back to Arduino UNO to make a confirmation call to the customer. The robot then asks the customer confirmation of the order and once the customer is verified Arduino UNO again sends a signal to the Arduino MEGA to lift up the desired payload compartment. When the customer takes the ordered item, the robot asks for the customer's satisfaction and further assistance. If the customer is satisfied, a final signal is sent from UNO to MEGA which drives the Robot back to the base. When after receiving one command there is another command, the robot performs an algorithm. The algorithm makes the robot decide to always deliver to the closest room first. If both the rooms are at equal distances from the base position of the robot, then it will first deliver to the room from where the first command was initiated. The movement of the robot for our system is based on time or delay. The time required for robot to move from its base to any room is calculated and that much delay is applied for the motors to be turned ON.

\section{Simulation}

Programming is performed using Arduino software (IDE) and with the help of different libraries for different modules like GPRS shield, TFT touchscreen etc.

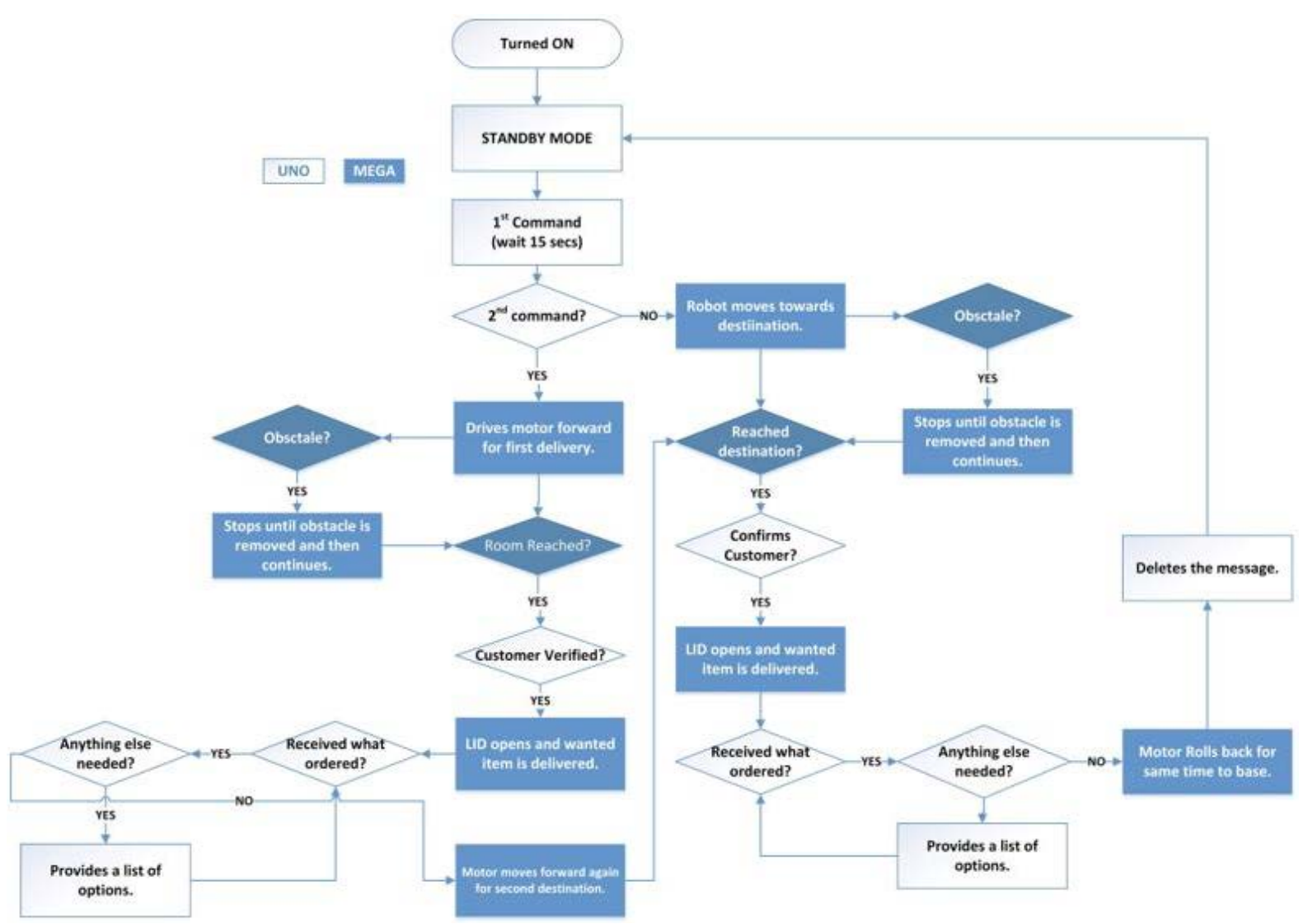

Figure 2. Flow diagram of the robot. 
Communication between the two Arduinos was done using the I2C protocol. The Inter-Integrated Circuit $\left(\mathrm{I}^{2} \mathrm{C}\right)$ Protocol is a protocol which allows multiple "Slave" digital integrated circuits to communicate with one "Master" chip. It is similar to Serial Peripheral Interface (SPI). It can only be used for short range communication within a single device. It only requires two signal wires to exchange information. The Master in our system is the Arduino UNO and MEGA the Slave.

The commands are sent in text format and in the following order: \#a/b (payload type) 1/2/3 (the last digit of room number). For e.g. \#a1 i.e. payload type "a" which can be water, ordered from room 101. \#b3 i.e. payload type "b" which can be towels ordered from room 103.

\subsection{Software Simulation}

The motors are attached with Arduino MEGA so when a command is received by GPRS Shield which is attached to Arduino UNO, it has to send a signal to MEGA to run the motors toward the following room. The following Characters are sent from UNO to Mega for the following purposes:

1. "A" to move from base to room $101^{*}$.

2. "B" to move from base to room $102^{*}$.

3. "C" to move from base to room $103^{*}$.

4. "D" to move from room 101 to room $102 *$.

5. "E" to move from room 101 to room $103^{*}$.

6. "F" to move from room 102 to room 103 ".

7. "G" to move from room 101 to base.

8. "H" to move from room 102 to base.

9. "I" to move from room 103 to base.

10. "e" to lift payload type A scissor lift.

11. "f" to lift payload type B scissor lift.

"Whenever these tasks are completed MEGA has to send a signal back to UNO, which is " 1 " in all cases. When UNO receives the signal it makes a call to the following room confirming the service has arrived.

\subsection{Touch Screen Interface}

Figure 3(a) shows the STANDBY MENU of the robot. If CALLS is pressed, the next menu (Figure 3(b)) shows up. At this point, the customer can select to make a call. Figure 3(c) shows a command being received by the Robot and Figure 3(d) shows the confirmation menu where the customer needs to press YES if he has made any order. Figure 3(f) shows the extended support provided by the robot to the customer where the customer can make any of the following choices if needed. If TOWEL or WATER is pressed the robot opens the lid again and delivers the required payload. If FOOD, ROOM CLEANING or OPERATOR is pressed, the robot makes a phone call to the related service.

\subsection{Final Prototype}

Figure 4 is displaying the final product and also showing the robot delivering a product to a customer.

\section{Conclusion and Future Work}

The prototype was designed as a final year project by four undergraduate students. It was tested on a scenario

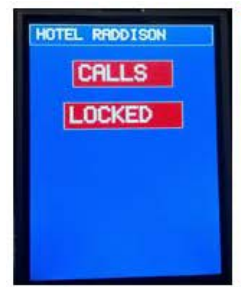

(a)

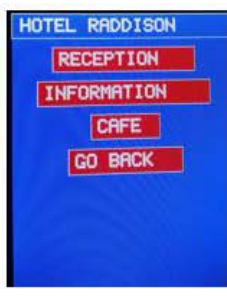

(b)

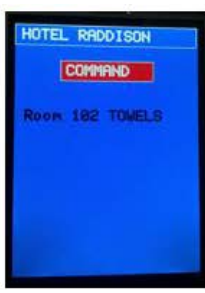

(c)

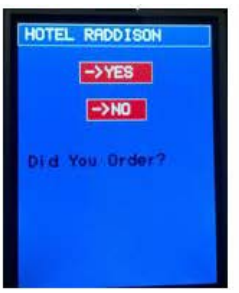

(d)

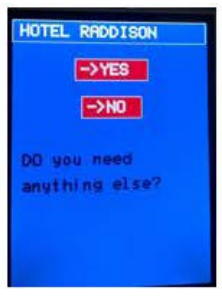

(e)

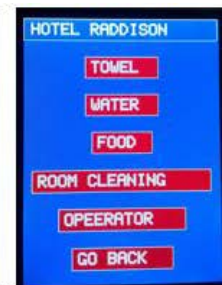

(f)

Figure 3. Touch display options. 


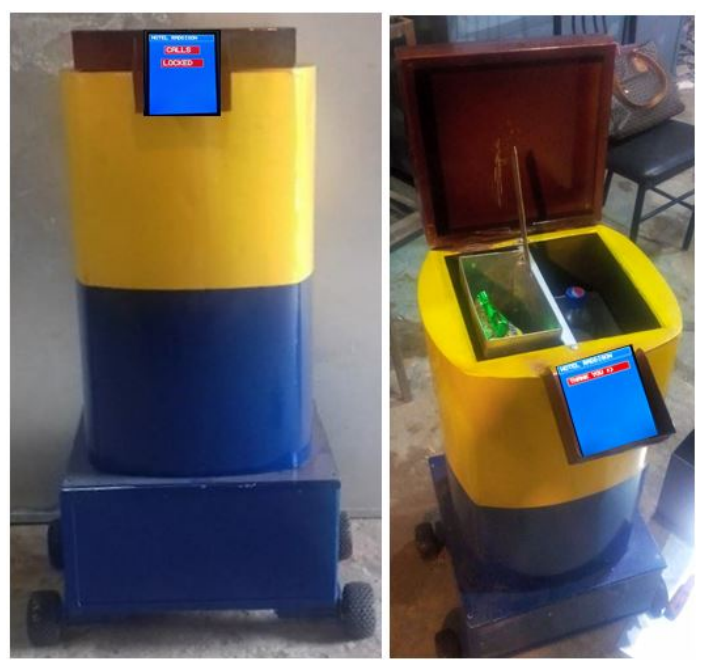

Figure 4. Complete robot (left) and robot delivering a payload to a customer (right).

with three rooms in a row and it had successfully delivered the ordered items in due time for both single and multiple commands.

In the future, this algorithm can be improved and modified to make the robot handle more than two commands in a hotel with a much more complex room distribution structure. Image sensing technology can be added to it to increase its accuracy in finding the rooms and detecting any obstacle. Hands can be added to it to deliver a payload to a customer, which could make it a much friendlier appearing humanoid.

\section{References}

[1] Rhodes, M. (2014) Meet the Company Designing Robot Bellhops for Hotel. http://www.wired.com/2014/08/meet-the-company-designing-robot-bellhops-for-hotels/

[2] Korthsha, M. (2014) The Value of Robotic Room Service Industry View. http://www.softwareadvice.com//hotel-management/industtryview/robotic-service-report-2014/

[3] SIMCom, A Company of SIM TECH: SIM 900 AT Command Manual_V1.11. www.sim.com/wm 